\title{
ON THE STABILITY OF SOME PROPERTIES OF PARTIAL ALGEBRAS UNDER POWERS
}

\author{
N. Chaisansuk ${ }^{*}$ - S. Leeratanavalee* $*$ J. Šlapal** \\ (Communicated by Jiři Rachůnek)
}

\begin{abstract}
In the paper, we study eight properties of partial algebras most of which are related to diagonality. For each of the properties, we give sufficient conditions under which this property is preserved by powers of partial algebras.

(C) 2014

Mathematical Institute

Slovak Academy of Sciences
\end{abstract}

\section{Introduction}

In his pioneering paper [1], G. Birkhoff introduced the operation of a cardinal power of partially ordered sets and showed the validity of the first exponential law for the operation, i.e., the law $\left(A^{B}\right)^{C} \cong A^{B \times C}$. In [6], J. Šlapal extended the concept of a power from partially ordered sets onto arbitrary $n$-ary relational systems and studied its behavior. The same author introduced and studied powers of $n$-ary algebras in 7 and also powers of partial algebras in [8]. He showed the importance of idempotency, diagonality (in the sense of [5]), mediality (in the sense of [3]), and commutativity (in the sense of [4]) of algebras and partial algebras when studying their powers. Moreover, in [9], J. Šlapal defined and studied the following eight properties of partial algebras: idempotency, diagonality, commutativity, strong diagonality, weak diagonality, local diagonality, local antidiagonality, and weak local antidiagonality. The aim of this paper

2010 Mathematics Subject Classification: Primary 08A05, 08A30, 08A55.

Keywords: partial algebra, reflexive, diagonal and commutative partial algebras, power of partial algebras.

This research was supported by the Royal Golden Jubilee Ph.D. Program of the Thailand Research Fund., the Graduate School and the Faculty of Science, Chiang Mai University, Thailand.

The third author acknowledges a support from the Brno University of Technology, project No. FSI-S-11-3. 


\section{N. CHAISANSUK - S. LEERATANAVALEE - J. ŠLAPAL}

is to give sufficient conditions for universal partial algebras under which these properties are preserved by exponentiating the algebras and under which the exponentiation satisfies the first exponential law.

\section{Partial algebras}

For the basic concepts used concerning partial algebras we refer to 2 . Let $\Omega$ be a nonempty set. A family $\tau=\left(K_{\lambda} ; \lambda \in \Omega\right)$ of sets will be called a type. By a partial algebra of type $\tau$ we understand a pair $\mathbf{G}=\left\langle G,\left(p_{\lambda} ; \lambda \in \Omega\right)\right\rangle$ where $G$ is a nonempty set, the so-called underlying set of $\mathbf{G}$, and $p_{\lambda}$ is a $K_{\lambda}$-ary partial operation on $G$, i.e., a map $p_{\lambda}: D_{p_{\lambda}} \rightarrow G$ where $D_{p_{\lambda}} \subseteq G^{K_{\lambda}}$, for each $\lambda \in \Omega$. The set $D_{p_{\lambda}}$ is called the domain of $p_{\lambda}$. If $K_{\lambda}$ is finite, then $p_{\lambda}$ is said to be finitary. Throughout the paper, the type $\tau$ of any partial algebra $\left\langle G,\left(p_{\lambda} ; \lambda \in \Omega\right)\right\rangle$ is understood to be the family denoted by $\left(K_{\lambda} ; \lambda \in \Omega\right)$. The underlying set of a partial algebra $\mathbf{G}$ will be denoted by $|\mathbf{G}|$. Of course, given a partial algebra $\left\langle G,\left(p_{\lambda} ; \lambda \in \Omega\right)\right\rangle$, when writing $p_{\lambda}\left(x_{i} ; i \in K_{\lambda}\right)=x$ (for some $x \in G$ ), we automatically mean that $p_{\lambda}\left(x_{i} ; i \in K_{\lambda}\right)$ is defined, i.e., that $\left(x_{i} ; i \in K_{\lambda}\right) \in D_{p_{\lambda}}$.

Let $\mathbf{H}=\left\langle H,\left(q_{\lambda} ; \lambda \in \Omega\right)\right\rangle, \mathbf{G}=\left\langle G,\left(p_{\lambda} ; \lambda \in \Omega\right)\right\rangle$ be a pair of partial algebras of type $\tau$. Then $\mathbf{G}$ is called a partial subalgebra of $\mathbf{H}$ provided that $G \subseteq H$ and, for each $\lambda \in \Omega$, whenever $\left(x_{i} ; i \in K_{\lambda}\right) \in G^{K_{\lambda}}$ and $x \in H$,

$$
p_{\lambda}\left(x_{i} ; i \in K_{\lambda}\right)=x \quad \Longleftrightarrow \quad q_{\lambda}\left(x_{i} ; i \in K_{\lambda}\right)=x
$$

(note that $D_{p_{\lambda}}=D_{q_{\lambda}} \cap G^{K_{\lambda}}$ ). A map $f: H \rightarrow G$ is called a homomorphism of $\mathbf{H}$ into $\mathbf{G}$ if, for each $\lambda \in \Omega, q_{\lambda}\left(x_{i} ; i \in K_{\lambda}\right)=x$ implies $p_{\lambda}\left(f\left(x_{i}\right) ; i \in K_{\lambda}\right)=f(x)$. The set of all homomorphisms of $\mathbf{H}$ into $\mathbf{G}$ will be denoted by $\operatorname{Hom}(\mathbf{H}, \mathbf{G})$.

The direct product of a family $\mathbf{G}_{i}=\left\langle G_{i},\left(p_{i_{\lambda}} ; \lambda \in \Omega\right)\right\rangle, i \in I$ of partial algebras of type $\tau$ is the partial algebra $\prod_{i \in I} \mathbf{G}_{i}=\left\langle\prod_{i \in I} G_{i},\left(q_{\lambda} ; \lambda \in \Omega\right)\right\rangle$ where $\prod_{i \in I} G_{i}$ denotes the cartesian product of sets and, for any $\lambda \in \Omega$, any $\left(f_{k}\right.$; $\left.k \in K_{\lambda}\right) \in\left(\prod_{i \in I} G_{i}\right)^{K_{\lambda}}$ and any $f \in \prod_{i \in I} G_{i}, q_{\lambda}\left(f_{k} ; k \in K_{\lambda}\right)=f$ if and only if $p_{i_{\lambda}}\left(f_{k}(i) ; k \in K_{\lambda}\right)=f(i)$ for each $i \in I$. If $\mathbf{G}_{i}=\mathbf{G}$ for every $i \in I$, then we write $\mathbf{G}^{I}$ instead of $\prod_{i \in I} \mathbf{G}_{i}$.

Given a partial algebra $\left\langle G,\left(p_{\lambda} ; \lambda \in \Omega\right)\right\rangle$ and $\lambda \in \Omega$, an element $x \in G$ is said to be a $p_{\lambda}$-idempotent if $p_{\lambda}\left(x_{i} ; i \in K_{\lambda}\right)=x$ whenever $x_{i}=x$ for each $i \in K_{\lambda}$.

Let $G, K, L$ be sets. By a $K \times L$-matrix $M$ over $G$ we understand any map $M: K \times L \rightarrow G$, i.e., $M=\left(x_{i j} ; i \in K, j \in L\right)$ where $x_{i j} \in G$ whenever $i \in K$ 
and $j \in L$. We denote by $M^{T}$ the transposed matrix to $M$, i.e., the matrix $M^{T}=\left(x_{j i} ; j \in L, i \in K\right)$ over $G$.

Let $x \in G$ and let $M=\left(x_{i j} ; i \in K, j \in L\right)$ be a $K \times L$-matrix $M$ over $G$. Then $M$ is said to be $x$-constant provided that $x_{i j}=x$ whenever $i \in K$ and $j \in L$, and it is said to have $x$-constant diagonal if $K=L$ and $x_{i i}=x$ for all $i \in K$. Let $q$ be an $L$-ary partial operation on $G$. Then $M$ is said to be $q$-operational provided that $\left(x_{i j} ; j \in L\right) \in D_{q}$ for each $i \in K$. Let $p$ be a $K$-ary partial operation on $G$. Then $M$ is said to be $p q$-operational provided that it is $q$-operational and $\left(q\left(x_{i j} ; j \in L\right) ; i \in K\right) \in D_{p}$; we put $p q(M)=p\left(q\left(x_{i j} ; j \in L\right) ; i \in K\right)$. Finally, $M$ is said to be diagonally p-operational provided that $K=L$ and $\left(x_{i i} ; i \in K\right) \in D_{p}$; we put $\Delta_{p}(M)=p\left(x_{i i} ; i \in K\right)$.

Definition 2.1. (9] A partial algebra $\left\langle G,\left(p_{\lambda} ; \lambda \in \Omega\right)\right\rangle$ of type $\tau$ is called

(1) idempotent if, for each $\lambda \in \Omega$, every element $x \in G$ is a $p_{\lambda}$-idempotent.

(2) commutative if, for any $\lambda, \mu \in \Omega$ and any $p_{\lambda} p_{\mu}$-operational $K_{\lambda} \times K_{\mu}$-matrix $M$ over $G$ such that $M^{T}$ is $p_{\lambda}$-operational, $M^{T}$ is $p_{\mu} p_{\lambda}$-operational with $p_{\lambda} p_{\mu}(M)=p_{\mu} p_{\lambda}\left(M^{T}\right)$,

(3) diagonal if, for any $\lambda \in \Omega$, every $p_{\lambda} p_{\lambda}$-operational $K_{\lambda} \times K_{\lambda}$ matrix $M$ over $G$ is diagonally $p_{\lambda}$-operational with $p_{\lambda} p_{\lambda}(M)=\Delta_{p_{\lambda}}(M)$,

(4) strongly diagonal if, for any $\lambda \in \Omega$ and any $x \in G$, any $p_{\lambda^{-o p e r a t i o n a l}}$ $K_{\lambda} \times K_{\lambda}$ matrix $M$ over $G$ is $p_{\lambda} p_{\lambda}$-operational with $p_{\lambda} p_{\lambda}(M)=x$ if and only if it is diagonally $p_{\lambda}$-operational with $\Delta_{p_{\lambda}}(M)=\mathrm{x}$,

(5) weakly diagonal if, for any $\lambda \in \Omega$ and any $p_{\lambda} p_{\lambda}$-operational $K_{\lambda} \times K_{\lambda}$ matrix $M$ over $G$, whenever $M^{T}$ is $p_{\lambda} p_{\lambda}$-operational with $p_{\lambda} p_{\lambda}(M)=p_{\lambda} p_{\lambda}\left(M^{T}\right)$, $M$ is diagonally $p_{\lambda}$-operational with $p_{\lambda} p_{\lambda}(M)=\Delta_{p_{\lambda}}(M)$,

(6) locally diagonal if, for any $\lambda \in \Omega$ and any $x \in G$, every $p_{\lambda}$-operational $K_{\lambda} \times K_{\lambda}$ matrix $M$ over $G$ with $x$-constant diagonal is $p_{\lambda} p_{\lambda}$-operational with $p_{\lambda} p_{\lambda}(M)=x$,

(7) locally antidiagonal if, for any $\lambda \in \Omega$, any $x \in G$ and any $p_{\lambda} p_{\lambda}$-operational $K_{\lambda} \times K_{\lambda}$ matrix $M$ over $G$ with $x$-constant diagonal, $p_{\lambda} p_{\lambda}(M)=x$ implies that $M$ is $x$-constant,

(8) weakly locally antidiagonal if, for any $\lambda \in \Omega$, any $x \in G$ and any $p_{\lambda} p_{\lambda \text {-oper- }}$ ational $K_{\lambda} \times K_{\lambda}$ matrix $M$ over $G$ with $x$-constant diagonal such that $M^{T}$ is $p_{\lambda} p_{\lambda}$-operational too, $p_{\lambda} p_{\lambda}(M)=p_{\lambda} p_{\lambda}\left(M^{T}\right)=x$ implies that $M$ is $x$-constant.

Clearly, strong diagonality implies diagonality, diagonality implies weak diagonality and local antidiagonality implies weak local antidiagonality. If $\mathbf{G}$ is idempotent, then strong diagonality implies local diagonality. 


\section{N. CHAISANSUK - S. LEERATANAVALEE - J. ŠLAPAL}

Example 2.2. Let $G$ be a set and $\rho$ be a binary relation on $G$. Let $p$ be the binary partial operation on $G$ given by

$$
D_{p}=\rho \quad \text { and } \quad p(a, b)=a \quad \text { whenever }(a, b) \in D_{p} .
$$

Let $q$ be the binary partial operation on $G$ dual to $p$, i.e., given by

$$
D_{q}=\rho \quad \text { and } \quad q(c, d)=d \quad \text { whenever }(c, d) \in D_{q} .
$$

Then $\langle G, p, q\rangle$ is a partial algebra of type $(2,2)$ and we have:

(a) $\langle G, p, q\rangle$ is commutative,

(b) $\rho$ is reflexive if and only if $\langle G, p, q\rangle$ is idempotent,

(c) $\rho$ is transitive if and only if $\langle G, p, q\rangle$ is diagonal,

(d) $\langle G, p, q\rangle$ is locally diagonal if $\rho$ is symmetric,

(e) $\langle G, p, q\rangle$ is strongly diagonal if $\rho$ is transitive and symmetric,

(f) $\rho$ is antisymmetric if and only if $\langle G, p, q\rangle$ is weakly locally antidiagonal.

Example 2.3. Let $(G, p)$ be a partial rectangular band, i.e., a partial algebra of type (2) such that there are sets $X, Y$ with $G=X \times Y$ and $p$ is the binary partial operation on $G$ given by

$$
D_{p}=\left\{\left(\left(a_{1}, b_{1}\right),\left(a_{2}, b_{2}\right)\right) \in(X \times Y)^{2}: b_{1}=a_{2}\right\}
$$

and

$$
p\left(\left(a_{1}, b_{1}\right),\left(a_{2}, b_{2}\right)\right)=\left(a_{1}, b_{2}\right) \quad \text { whenever } \quad\left(\left(a_{1}, b_{1}\right),\left(a_{2}, b_{2}\right)\right) \in D_{p} .
$$

Then $(G, p)$ is a weakly diagonal partial algebra.

Example 2.4. Let $X$ be a set and let $p$ be a binary partial operation on the power set $\mathcal{P}(X)$ of $X$ given by

$$
\begin{aligned}
(A, B) \in D_{p} \Longleftrightarrow & {[A=B \text { or }(A \neq \emptyset \& B \neq \emptyset \& A \cap B=\emptyset)] } \\
& \text { and then } p(A, B)=A \cap B .
\end{aligned}
$$

Then $(\mathcal{P}(X), p)$ is a locally antidiagonal partial algebra.

Let $\mathbf{H}=\left\langle H,\left(q_{\lambda} ; \lambda \in \Omega\right)\right\rangle$ and $\mathbf{G}=\left\langle G,\left(p_{\lambda} ; \lambda \in \Omega\right)\right\rangle$ be partial algebras of the same type, let $\lambda \in \Omega$ and let $\left(f_{i} ; i \in K_{\lambda}\right) \in(\operatorname{Hom}(\mathbf{H}, \mathbf{G}))^{K_{\lambda}}$. Let $f \in \operatorname{Hom}(\mathbf{H}, \mathbf{G})$ be a homomorphism such that, for all $\left(x_{i} ; i \in K_{\lambda}\right) \in H^{K_{\lambda}}$ and all $x \in H, q_{\lambda}\left(x_{i} ; i \in K_{\lambda}\right)=x$ implies $p_{\lambda}\left(f_{i}\left(x_{i}\right) ; i \in K_{\lambda}\right)=f(x)$. Then $f$ need not be a unique homomorphism of $\mathbf{H}$ into $\mathbf{G}$ having this property - see the following example.

Example 2.5. Let $\mathbf{H}=(H, q), \mathbf{G}=(G, p)$ be partial algebras of type (2) where $H=\{1,2\}, G=\{a, b\}$ and $p, q$ are given by the tables 


\begin{tabular}{|l|ll|}
\hline$q$ & 1 & 2 \\
\hline 1 & 2 & 2 \\
2 & 2 & 2 \\
\hline
\end{tabular}

and

\begin{tabular}{|l|ll|}
\hline$p$ & $a$ & $b$ \\
\hline$a$ & $a$ & $a$ \\
$b$ & $a$ & $a$ \\
\hline
\end{tabular}

Let $f: H \rightarrow G$ be the map given by $f(1)=a, f(2)=a$ and $g: H \rightarrow G$ be the map given by $g(1)=b, g(2)=a$. Then $f, g \in \operatorname{Hom}(\mathbf{H}, \mathbf{G})$ and, for any $x, y, z \in\{1,2\}, q(x, y)=z$ implies $p(f(x), f(y))=f(z)$ and $p(f(x), f(y))=g(z)$. But $f \neq g$.

Lemma 2.6. Let $\mathbf{H}=\left\langle H,\left(q_{\lambda} ; \lambda \in \Omega\right)\right\rangle$, $\mathbf{G}=\left\langle G,\left(p_{\lambda} ; \lambda \in \Omega\right)\right\rangle$ be partial algebras of the same type, $\mathbf{H}$ idempotent, and let $\lambda \in \Omega$ and $\left(f_{i} ; i \in K_{\lambda}\right) \in$ $(\operatorname{Hom}(\mathbf{H}, \mathbf{G}))^{K_{\lambda}}$. If $f \in \operatorname{Hom}(\mathbf{H}, \mathbf{G})$ is a homomorphism such that, for all $\left(x_{i} ; i \in K_{\lambda}\right) \in H^{K_{\lambda}}$ and all $x \in H$,

$$
q_{\lambda}\left(x_{i} ; i \in K_{\lambda}\right)=x \Longrightarrow p_{\lambda}\left(f_{i}\left(x_{i}\right) ; i \in K_{\lambda}\right)=f(x),
$$

then $f$ is uniquely determined.

Proof. Let $f, g \in \operatorname{Hom}(\mathbf{H}, \mathbf{G})$ be homomorphisms such that, for all $\left(x_{i}\right.$; $\left.i \in K_{\lambda}\right) \in H^{K_{\lambda}}$ and all $x \in H, q_{\lambda}\left(x_{i} ; i \in K_{\lambda}\right)=x$ implies both $p_{\lambda}\left(f_{i}\left(x_{i}\right)\right.$; $\left.i \in K_{\lambda}\right)=f(x)$ and $p_{\lambda}\left(f_{i}\left(x_{i}\right) ; i \in K_{\lambda}\right)=g(x)$. Let $x \in H$. As $\mathbf{H}$ is idempotent, putting $x_{i}=x$ for each $i \in K_{\lambda}$, we get $q_{\lambda}\left(x_{i} ; i \in K_{\lambda}\right)=x$, $p_{\lambda}\left(f_{i}(x) ; i \in K_{\lambda}\right)=f(x)$ and $p_{\lambda}\left(f_{i}(x) ; \in K_{\lambda}\right)=g(x)$. Hence, $f=g$.

\section{Stability of properties of partial algebras under powers}

Definition 3.1. Let $\mathbf{H}=\left\langle H,\left(q_{\lambda} ; \lambda \in \Omega\right)\right\rangle$ and $\mathbf{G}=\left\langle G,\left(p_{\lambda} ; \lambda \in \Omega\right)\right\rangle$ be partial algebras of the same type $\tau$. The power of $\mathbf{G}$ and $\mathbf{H}$ is the partial algebra $\mathbf{G}^{\mathbf{H}}=\left\langle\operatorname{Hom}(\mathbf{H}, \mathbf{G}),\left(r_{\lambda} ; \lambda \in \Omega\right)\right\rangle$ of type $\tau$ where, for any $\lambda \in \Omega$, any $\left(f_{i} ; i \in K_{\lambda}\right) \in(\operatorname{Hom}(\mathbf{H}, \mathbf{G}))^{K_{\lambda}}$ and any $f \in \operatorname{Hom}(\mathbf{H}, \mathbf{G}), r_{\lambda}\left(f_{i} ; i \in K_{\lambda}\right)=f$ if and only if $f$ is a unique homomorphism of $\mathbf{H}$ into $\mathbf{G}$ with the property that, for all $\left(x_{i} ; i \in K_{\lambda}\right) \in H^{K_{\lambda}}$ and all $x \in H, q_{\lambda}\left(x_{i} ; i \in K_{\lambda}\right)=x$ implies $p_{\lambda}\left(f_{i}\left(x_{i}\right) ; i \in K_{\lambda}\right)=f(x)$.

Lemma 2.6 and Definition 3.1 immediately result in

Corollary 3.2. Let $\mathbf{G}, \mathbf{H}$ be partial algebras of the same type. If $\mathbf{H}$ is idempotent, then the power $\mathbf{G}^{H}$ is idempotent, too. 


\section{N. CHAISANSUK - S. LEERATANAVALEE - J. ŠLAPAL}

Lemma 3.3. Let $\mathbf{G}=\left\langle G,\left(p_{\lambda} ; \lambda \in \Omega\right)\right\rangle, \mathbf{H}=\left\langle H,\left(q_{\lambda} ; \lambda \in \Omega\right)\right\rangle$ be partial algebras of the same type, $\mathbf{H}$ idempotent, and let $\mathbf{G}^{\mathbf{H}}=\left\langle\operatorname{Hom}(\mathbf{H}, \mathbf{G}),\left(r_{\lambda} ; \lambda \in \Omega\right)\right\rangle$. Let $\lambda, \mu \in \Omega$ and let $M=\left(f_{i j} ; i \in K_{\lambda}, j \in K_{\mu}\right)$ be a matrix over $\operatorname{Hom}(\mathbf{H}, \mathbf{G})$. If $M$ is $r_{\mu}$-operational with $r_{\mu}\left(f_{i j} ; j \in K_{\mu}\right)=f_{i}$ for each $i \in K_{\lambda}, r_{\lambda} r_{\mu}$-operational with $r_{\lambda} r_{\mu}(M)=f$ or diagonally $r_{\lambda}$-operational with $\Delta_{r_{\lambda}}(M)=f$, respectively, then, whenever $\left(y_{j} ; j \in K_{\mu}\right) \in D_{q_{\mu}}$ and $q_{\mu}\left(y_{j} ; j \in K_{\mu}\right)=y$, the matrix $M^{*}=\left(f_{i j}\left(y_{j}\right) ; i \in K_{\lambda}, j \in K_{\mu}\right)$ over $G$ is $p_{\mu}$-operational with $p_{\mu}\left(f_{i j}\left(y_{j}\right)\right.$; $\left.j \in K_{\mu}\right)=f_{i}(y)$ for each $i \in K_{\lambda}, p_{\lambda} p_{\mu}$-operational with $p_{\lambda} p_{\mu}\left(M^{*}\right)=f(y)$ or diagonally $p_{\lambda}$-operational with $\Delta_{p_{\lambda}}\left(M^{*}\right)=f(y)$, respectively.

Proof.

(1) Let $M$ be $r_{\mu}$-operational with $r_{\mu}\left(f_{i j} ; j \in K_{\mu}\right)=f_{i}$ for each $i \in K_{\lambda}$ and let $\left(y_{j} ; j \in K_{\mu}\right) \in D_{q_{\mu}}, q_{\mu}\left(y_{j} ; j \in K_{\mu}\right)=y$. Then it follows immediately from Definition 3.1 that $M^{*}$ is $p_{\mu}$-operational with $p_{\mu}\left(f_{i j}\left(y_{j}\right) ; j \in K_{\mu}\right)=f_{i}(y)$ for each $i \in K_{\lambda}$.

(2) Let $M$ be $r_{\lambda} r_{\mu}$-operational with $r_{\lambda} r_{\mu}(M)=f$ and put $r_{\mu}\left(f_{i j} ; j \in K_{\mu}\right)=f_{i}$ for each $i \in K_{\lambda}$. Let $\left(y_{j} ; j \in K_{\mu}\right) \in D_{q_{\mu}}, q_{\mu}\left(y_{j} ; j \in K_{\mu}\right)=y$. By (1), $M^{*}$ is $p_{\mu}$-operational with $p_{\mu}\left(f_{i j}\left(y_{j}\right) ; j \in K_{\mu}\right)=f_{i}(y)$ for each $i \in K_{\lambda}$. Thus,

$$
p_{\lambda} p_{\mu}\left(M^{*}\right)=p_{\lambda}\left(p_{\mu}\left(f_{i j}\left(y_{j}\right)\right) ; j \in K_{\mu}, i \in K_{\lambda}\right)=p_{\lambda}\left(f_{i}(y) ; i \in K_{\lambda}\right) .
$$

As $\mathbf{H}$ is idempotent, we get $p_{\lambda}\left(f_{i}(y) ; i \in K_{\lambda}\right)=f(y)$. Consequently, $M^{*}$ is $p_{\lambda} p_{\mu}$-operational with $p_{\lambda} p_{\mu}\left(M^{*}\right)=f(y)$.

(3) Let $M$ be diagonally $r_{\lambda^{-}}$operational with $\Delta_{r_{\lambda}}(M)=f$, i.e., let $r_{\lambda}\left(f_{i i}\right.$; $\left.i \in K_{\lambda}\right)=f$. Let $\left(y_{j} ; j \in K_{\mu}\right) \in D_{q_{\mu}}, q_{\mu}\left(y_{j} ; j \in K_{\mu}\right)=y$. Then

$$
\Delta_{p_{\lambda}}\left(M^{*}\right)=p_{\lambda}\left(f_{i i}\left(y_{i}\right) ; i \in K_{\lambda}\right)=f(y) .
$$

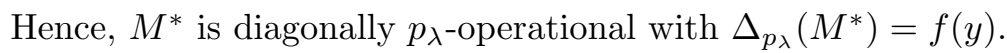

Using Lemma 3.3, one can easily prove the following theorem.

TheOREM 3.4. Let $\mathbf{G}, \mathbf{H}$ be partial algebras of the same type, $\mathbf{H}$ idempotent. If $\mathbf{G}$ is commutative, diagonal, strongly diagonal or weakly diagonal, respectively, then so is $\mathbf{G}^{\mathbf{H}}$.

Lemma 3.5. Let $\mathbf{G}=\left\langle G,\left(p_{\lambda} ; \lambda \in \Omega\right)\right\rangle$ be an idempotent, locally diagonal partial algebra and let $\lambda \in \Omega$. If $\left(x_{i} ; i \in K_{\lambda}\right),\left(y_{i} ; i \in K_{\lambda}\right) \in G^{K_{\lambda}}, p_{\lambda}\left(y_{i} ; i \in K_{\lambda}\right)=y$ and there exists $i_{0} \in K_{\lambda}$ such that $x_{i_{0}}=y$ and $x_{i}=y_{i_{0}}$ for each $i \in K_{\lambda}-\left\{i_{0}\right\}$, then $p_{\lambda}\left(x_{i} ; i \in K_{\lambda}\right)=y_{i_{0}}$.

Proof. Let $\left(x_{i} ; i \in K_{\lambda}\right),\left(y_{i} ; i \in K_{\lambda}\right) \in G^{K_{\lambda}}, p_{\lambda}\left(y_{i} ; i \in K_{\lambda}\right)=y$ and let $i_{0} \in K_{\lambda}$ be an element such that $x_{i_{0}}=y$ and $x_{i}=y_{i_{0}}$ for each $i \in K_{\lambda}-\left\{i_{0}\right\}$. Let $M=\left(a_{i j} ; i \in K_{\lambda}, j \in K_{\lambda}\right)$ be a $K_{\lambda} \times K_{\lambda}$-matrix over $\mathbf{G}$ such that $a_{i j}=y_{i_{0}}$ 
for each $i \in K_{\lambda}-\left\{i_{0}\right\}$ and $a_{i_{0} j}=y_{j}$ for each $j \in K_{\lambda}$. Since $\mathbf{G}$ is idempotent, $M$ is $p_{\lambda}$-operational with $y_{i_{0}}$-constant diagonal. As $\mathbf{G}$ is locally diagonal, we have

$$
p_{\lambda} p_{\lambda}(M)=p_{\lambda}\left(p_{\lambda}\left(a_{i j} ; j \in K_{\lambda}\right) ; i \in K_{\lambda}\right)=y_{i_{0}} .
$$

Thus, $p_{\lambda}\left(x_{i} ; i \in K_{\lambda}\right)=y_{i_{0}}$ because $x_{i}=p_{\lambda}\left(a_{i j} ; j \in K_{\lambda}\right)$ for every $i \in K_{\lambda}$.

TheOREm 3.6. Let $\mathbf{G}, \mathbf{H}$ be partial algebras of the same type, $\mathbf{H}$ idempotent and locally diagonal. If $\mathbf{G}$ is locally diagonal, then so is $\mathbf{G}^{\mathbf{H}}$.

Proof. Let $\mathbf{H}=\left\langle H,\left(q_{\lambda} ; \lambda \in \Omega\right)\right\rangle, \mathbf{G}=\left\langle G,\left(p_{\lambda} ; \lambda \in \Omega\right)\right\rangle, \mathbf{G}^{\mathbf{H}}=\langle\operatorname{Hom}(\mathbf{H}, \mathbf{G})$, $\left.\left(r_{\lambda} ; \lambda \in \Omega\right)\right\rangle$ and let $\mathbf{G}$ be locally diagonal. Let $\lambda \in \Omega$ and $f \in \operatorname{Hom}(\mathbf{H}, \mathbf{G})$. Let $M=\left(f_{i j} ; i \in K_{\lambda}, j \in K_{\lambda}\right)$ be an $r_{\lambda}$-operational matrix over $\operatorname{Hom}(\mathbf{H}, \mathbf{G})$ with $f$-constant diagonal. Put $r_{\lambda}\left(f_{i j} ; j \in K_{\lambda}\right)=f_{i}$ for each $i \in K_{\lambda}$. Let $\left(y_{i} ; i \in K_{\lambda}\right) \in D_{q_{\lambda}}, q_{\lambda}\left(y_{i} ; i \in K_{\lambda}\right)=y$ and, for every $i \in K_{\lambda}$, put $a_{i i}=y$ and $a_{i j}=y_{i}$ for each $j \in K_{\lambda}-\{i\}$. As $\mathbf{H}$ is idempotent and locally diagonal, we have $q_{\lambda}\left(a_{i j} ; j \in K_{\lambda}\right)=y_{i}$ for every $i \in K_{\lambda}$ by Lemma 3.5. Thus, $p_{\lambda}\left(f_{i j}\left(a_{i j}\right)\right.$; $\left.j \in K_{\lambda}\right)=f_{i}\left(y_{i}\right)$ for every $i \in K_{\lambda}$. Put $M^{*}=\left(f_{i j}\left(a_{i j}\right) ; i \in K_{\lambda}, j \in K_{\lambda}\right)$. Then $M^{*}$ is a $p_{\lambda}$-operational $K_{\lambda} \times K_{\lambda}$-matrix over $\mathbf{G}$ with $f(y)$-constant diagonal. Since $\mathbf{G}$ is locally diagonal, we have $p_{\lambda} p_{\lambda}\left(M^{*}\right)=f(y)$. Therefore,

$$
p_{\lambda}\left(f_{i}\left(y_{i}\right) ; i \in K_{\lambda}\right)=f(y) .
$$

Since $\mathbf{H}$ is idempotent, $f$ is uniquely determined. Hence, $r_{\lambda}\left(f_{i} ; i \in K_{\lambda}\right)=$ $r_{\lambda} r_{\lambda}(M)=f$.

Theorem 3.7. Let $\mathbf{G}, \mathbf{H}$ be partial algebras of the same type. $\mathbf{H}$ idempotent. If $\mathbf{G}$ is locally antidiagonal or weakly locally antidiagonal, respectively, then so is $\mathbf{G}^{\mathbf{H}}$.

Proof. Let $\mathbf{G}=\left\langle G,\left(p_{\lambda} ; \lambda \in \Omega\right)\right\rangle, \mathbf{H}=\left\langle H,\left(q_{\lambda} ; \lambda \in \Omega\right)\right\rangle$ be partial algebras of the same type, $\mathbf{H}$ idempotent, and let $\mathbf{G}^{\mathbf{H}}=\left\langle\operatorname{Hom}(\mathbf{H}, \mathbf{G}),\left(r_{\lambda} ; \lambda \in \Omega\right)\right\rangle$. Let $\lambda \in \Omega$ and $f \in \operatorname{Hom}(\mathbf{H}, \mathbf{G})$. Let $M=\left(f_{i j} ; i \in K_{\lambda}, j \in K_{\lambda}\right)$ be an $r_{\lambda} r_{\lambda}$-operational matrix over $\operatorname{Hom}(\mathbf{H}, \mathbf{G})$ with $f$-constant diagonal and let $y \in H$. Since $\mathbf{H}$ is idempotent, $M^{*}=\left(f_{i j}(x) ; i \in K_{\lambda}, j \in K_{\lambda}\right)$ is a $p_{\lambda} p_{\lambda}$-operational matrix over $G$ by Lemma 3.3. It is also evident that $M^{*}$ has $f(y)$-constant diagonal. Using these facts, we easily get the statement.

Theorem 3.8. Let $\mathbf{G}, \mathbf{H}, \mathbf{K}$ be partial algebras of the same type. If $\mathbf{H}, \mathbf{K}$ are idempotent, then

$$
\left(\mathbf{G}^{\mathbf{H}}\right)^{\mathbf{K}} \cong \mathbf{G}^{\mathbf{H} \times \mathbf{K}} \text {. }
$$

Proof. Let

$$
\begin{array}{ll}
\mathbf{H}=\left\langle H,\left(q_{\lambda} ; \lambda \in \Omega\right)\right\rangle, & \mathbf{G}=\left\langle G,\left(p_{\lambda} ; \lambda \in \Omega\right)\right\rangle, \\
\mathbf{K}=\left\langle K,\left(s_{\lambda} ; \lambda \in \Omega\right)\right\rangle, & \mathbf{G}^{\mathbf{H}}=\left\langle\operatorname{Hom}(\mathbf{H}, \mathbf{G}),\left(R_{\lambda} ; \lambda \in \Omega\right)\right\rangle,
\end{array}
$$


$\mathbf{H} \times \mathbf{K}=\left\langle H \times K,\left(V_{\lambda} ; \lambda \in \Omega\right)\right\rangle, \quad\left(\mathbf{G}^{\mathbf{H}}\right)^{\mathbf{K}}=\left\langle\operatorname{Hom}(\mathbf{K}, \operatorname{Hom}(\mathbf{H}, \mathbf{G})),\left(T_{\lambda} ; \lambda \in \Omega\right)\right\rangle$, $\mathbf{G}^{\mathbf{H} \times \mathbf{K}}=\left\langle\operatorname{Hom}(\mathbf{H} \times \mathbf{K}, \mathbf{G}),\left(U_{\lambda} ; \lambda \in \Omega\right)\right\rangle$

and let $\mathbf{H}, \mathbf{K}$ be idempotent.

Let $\lambda \in \Omega$ and define a map $\varphi: \operatorname{Hom}(\mathbf{K}, \operatorname{Hom}(\mathbf{H}, \mathbf{G})) \rightarrow \operatorname{Hom}(\mathbf{H} \times \mathbf{K}, \mathbf{G})$ by $\varphi(g)(y, z)=g(z)(y)$ whenever $g \in \operatorname{Hom}(\mathbf{K}, \operatorname{Hom}(\mathbf{H}, \mathbf{G})), z \in K$ and $y \in H$. We will show first that the map $\varphi$ is well defined, i.e., that $\varphi(g) \in$ $\operatorname{Hom}(\mathbf{H} \times \mathbf{K}, \mathbf{G})$ whenever $g \in \operatorname{Hom}(\mathbf{K}, \operatorname{Hom}(\mathbf{H}, \mathbf{G}))$. For this account, let $g \in\left(\operatorname{Hom}(\mathbf{K}, \operatorname{Hom}(\mathbf{H}, \mathbf{G}))\right.$ and let $\left(\left(y_{i}, z_{i}\right) ; i \in K_{\lambda}\right) \in D_{V_{\lambda}}, V_{\lambda}\left(\left(y_{i}, z_{i}\right) ; i \in K_{\lambda}\right)$ $=(y, z)$. Then $q_{\lambda}\left(y_{i} ; i \in K_{\lambda}\right)=y$ and $s_{\lambda}\left(z_{i} ; i \in K_{\lambda}\right)=z$ and, since $g \in\left(\operatorname{Hom}(\mathbf{K}, \operatorname{Hom}(\mathbf{H}, \mathbf{G}))\right.$, we get $R_{\lambda}\left(g\left(z_{i}\right) ; i \in K_{\lambda}\right)=g(z)$. This implies

$$
p_{\lambda}\left(g\left(z_{i}\right)\left(y_{i}\right) ; i \in K_{\lambda}\right)=g(z)(y)
$$

and, therefore,

$$
p_{\lambda}\left(\varphi(g)\left(y_{i}, z_{i}\right) ; i \in K_{\lambda}\right)=p_{\lambda}\left(g\left(z_{i}\right)\left(y_{i}\right) ; i \in K_{\lambda}\right)=g(z)(y)=\varphi(g)(y, z) .
$$

Hence, $\varphi(g) \in \operatorname{Hom}(\mathbf{H} \times \mathbf{K}, \mathbf{G})$.

Further, define a map $\alpha: \operatorname{Hom}(\mathbf{H} \times \mathbf{K}, \mathbf{G}) \rightarrow \operatorname{Hom}(\mathbf{K}, \operatorname{Hom}(\mathbf{H}, \mathbf{G}))$ by $\alpha(h)(z)(y)=h(y, z)$ whenever $h \in \operatorname{Hom}(\mathbf{H} \times \mathbf{K}, \mathbf{G}), z \in K$ and $y \in H$. We will show that $\alpha$ is well defined, too.

Let $h \in \operatorname{Hom}(\mathbf{H} \times \mathbf{K}, \mathbf{G})$. To show that $\alpha(h)(z) \in \operatorname{Hom}(\mathbf{H}, \mathbf{G})$ for each $z \in K$, let $z \in K$ and $\left(y_{i} ; i \in K_{\lambda}\right) \in D_{q_{\lambda}}, q_{\lambda}\left(y_{i} ; i \in K_{\lambda}\right)=y$. Since $\mathbf{K}$ is idempotent, we have $V_{\lambda}\left(\left(y_{i}, z\right) ; i \in K_{\lambda}\right)=(y, z)$. From $h \in \operatorname{Hom}(\mathbf{H} \times \mathbf{K}, \mathbf{G})$ it follows that $p_{\lambda}\left(h\left(y_{i}, z\right) ; i \in K_{\lambda}\right)=h(y, z)$. Thus,

$$
p_{\lambda}\left(\alpha(h)(z)\left(y_{i}\right) ; i \in K_{\lambda}\right)=p_{\lambda}\left(h\left(y_{i}, z\right) ; i \in K_{\lambda}\right)=h(y, z)=\alpha(h)(z)(y),
$$

so that $\alpha(h)(z) \in \operatorname{Hom}(\mathbf{H}, \mathbf{G})$.

To show that $\alpha(h) \in \operatorname{Hom}(\mathbf{K}, \operatorname{Hom}(\mathbf{H}, \mathbf{G}))$, let $\left(z_{i} ; i \in K_{\lambda}\right) \in D_{s_{\lambda}}$, $s_{\lambda}\left(z_{i} ; i \in K_{\lambda}\right)=z$ and let $\left(y_{i} ; i \in K_{\lambda}\right) \in D_{q_{\lambda}}, q_{\lambda}\left(y_{i} ; i \in K_{\lambda}\right)=y$. Then $V_{\lambda}\left(\left(y_{i}, z_{i}\right) ; i \in K_{\lambda}\right)=(y, z)$. Since $h \in \operatorname{Hom}(\mathbf{H} \times \mathbf{K}, \mathbf{G})$, we get $p_{\lambda}\left(h\left(y_{i}, z_{i}\right)\right.$; $\left.i \in K_{\lambda}\right)=h(y, z)$. So,

$$
p_{\lambda}\left(\alpha(h)\left(z_{i}\right)\left(y_{i}\right) ; i \in K_{\lambda}\right)=p_{\lambda}\left(h\left(y_{i}, z_{i}\right) ; i \in K_{\lambda}\right)=h(y, z)=\alpha(h)(z)(y) .
$$

Since $\mathbf{H}$ is idempotent, $\alpha(h)(z)$ is uniquely determined. Consequently,

$$
R_{\lambda}\left(\alpha(h)\left(z_{i}\right) ; i \in K_{\lambda}\right)=\alpha(h)(z),
$$

so that $\alpha(h) \in \operatorname{Hom}(\mathbf{K}, \operatorname{Hom}(\mathbf{H}, \mathbf{G}))$.

Further, we have

$$
\varphi(\alpha(h))(y, z)=\alpha(h)(z)(y)=h(y, z) \quad \text { and } \quad \alpha(\varphi(g))(z)(y)=\varphi(g)(y, z)=g(z)(y)
$$

for all $h \in \operatorname{Hom}(\mathbf{H} \times \mathbf{K}, \mathbf{G}), g \in \operatorname{Hom}(\mathbf{K}, \operatorname{Hom}(\mathbf{H}, \mathbf{G})), y \in H$ and $z \in K$. Consequently, $\varphi$ and $\alpha$ are bijections inverse to each other. 
To show that $\varphi$ is a homomorphism, let $\left(g_{i} ; i \in K_{\lambda}\right) \in D_{T_{\lambda}}, T_{\lambda}\left(g_{i} ; i \in K_{\lambda}\right)$ $=g$ and $\left(\left(y_{i}, z_{i}\right) ; i \in K_{\lambda}\right) \in D_{V_{\lambda}}, V_{\lambda}\left(\left(y_{i}, z_{i}\right) ; i \in K_{\lambda}\right)=(y, z)$. Then $q_{\lambda}\left(y_{i} ; i \in K_{\lambda}\right)=y$ and $s_{\lambda}\left(z_{i} ; i \in K_{\lambda}\right)=z$. Hence, $R_{\lambda}\left(g_{i}\left(z_{i}\right) ; i \in K_{\lambda}\right)$ $=g(z)$ and, consequently, $p_{\lambda}\left(g_{i}\left(z_{i}\right)\left(y_{i}\right) ; i \in K_{\lambda}\right)=g(z)(y)$ and $g(z)$ is uniquely determined because $\mathbf{H} \times \mathbf{K}$ is idempotent. Therefore,

$$
p_{\lambda}\left(\varphi\left(g_{i}\right)\left(y_{i}, z_{i}\right) ; i \in K_{\lambda}\right)=p_{\lambda}\left(g_{i}\left(z_{i}\right)\left(y_{i}\right) ; i \in K_{\lambda}\right)=g(z)(y)=\varphi(g)(y, z)
$$

and $\varphi(g)$ is uniquely determined because $\mathbf{H} \times \mathbf{K}$ is idempotent. We have $U_{\lambda}\left(\varphi\left(g_{i}\right) ; i \in K_{\lambda}\right)=\varphi(g)$. Thus, $\varphi$ is a homomorphism.

To show that $\alpha$ is a homomorphism, let $\left(h_{i} ; i \in K_{\lambda}\right) \in D_{U_{\lambda}}, U_{\lambda}\left(h_{i} ; i \in K_{\lambda}\right)$ $=h,\left(z_{i} ; i \in K_{\lambda}\right) \in D_{s_{\lambda}}, s_{\lambda}\left(z_{i} ; i \in K_{\lambda}\right)=z$ and $\left(y_{i} ; i \in K_{\lambda}\right) \in D_{q_{\lambda}}$, $q_{\lambda}\left(y_{i} ; i \in K_{\lambda}\right)=y$. Then we have $V_{\lambda}\left(\left(y_{i}, z_{i}\right) ; i \in K_{\lambda}\right)=(y, z)$ and, consequently, $p_{\lambda}\left(h_{i}\left(y_{i}, z_{i}\right) ; i \in K_{\lambda}\right)=h(y, z)$. Therefore,

$$
p_{\lambda}\left(\alpha\left(h_{i}\right)\left(z_{i}\right)\left(y_{i}\right) ; i \in K_{\lambda}\right)=p_{\lambda}\left(h_{i}\left(y_{i}, z_{i}\right) ; i \in K_{\lambda}\right)=h(y, z)=\alpha(h)(z)(y)
$$

and $\alpha(h)(z)$ is uniquely determined because $\mathbf{H}$ is idempotent. Consequently, $R_{\lambda}\left(\alpha\left(h_{i}\right)\left(z_{i}\right) ; i \in K_{\lambda}\right)=\alpha(h)(z)$. The idempotency of $\mathbf{K}$ implies the uniqueness of $\alpha(h)$, hence $T_{\lambda}\left(\alpha\left(h_{i}\right) ; i \in K_{\lambda}\right)=\alpha(h)$. Therefore, $\alpha$ is a homomorphism.

We have shown that $\varphi: \operatorname{Hom}(\mathbf{K}, \operatorname{Hom}(\mathbf{H}, \mathbf{G})) \rightarrow \operatorname{Hom}(\mathbf{H} \times \mathbf{K}, \mathbf{G})$ is an isomorphism (with $\varphi^{-1}=\alpha$ ), i.e., $\left(\mathbf{G}^{\mathbf{H}}\right)^{\mathbf{K}} \cong \mathbf{G}^{\mathbf{H} \times \mathbf{K}}$.

TheOrem 3.9. Let $\mathbf{G}, \mathbf{H}$ be partial algebras of the same type. If $\mathbf{G}$ is commutative, then there exists a partial subalgebra $\mathbf{K}$ of the direct product $\mathbf{G}^{|\mathbf{H}|}$ such that $|\mathbf{K}|=\operatorname{Hom}(\mathbf{H}, \mathbf{G})$.

Proof. Let $\mathbf{H}=\left\langle H,\left(q_{\lambda} ; \lambda \in \Omega\right)\right\rangle, \mathbf{G}=\left\langle G,\left(p_{\lambda} ; \lambda \in \Omega\right)\right\rangle \mathbf{G}^{|\mathbf{H}|}$ $=\left\langle G^{H},\left(R_{\lambda} ; \lambda \in \Omega\right)\right\rangle$ and let $\mathbf{G}$ be commutative. Let $\lambda \in \Omega$, let $\left(f_{i} ; i \in K_{\lambda}\right)$ $\in D_{R_{\lambda}} \cap(\operatorname{Hom}(\mathbf{H}, \mathbf{G}))^{K_{\lambda}}, R_{\lambda}\left(f_{i} ; i \in K_{\lambda}\right)=f$. Let $\mu \in \Omega$ and let $\left(y_{j} ;\right.$ $\left.j \in K_{\mu}\right) \in D_{q_{\mu}}, q_{\mu}\left(y_{j} ; j \in K_{\mu}\right)=y$. Consider the $K_{\lambda} \times K_{\mu}$-matrix $M=$ $\left(f_{i}\left(y_{j}\right) ; i \in K_{\lambda}, j \in K_{\mu}\right)$ over G. Then, for each $i \in K_{\lambda}, p_{\lambda}\left(f_{i}\left(y_{j}\right)\right.$; $\left.j \in K_{\mu}\right)=f_{i}(y)$ because $f_{i} \in \operatorname{Hom}(\mathbf{H}, \mathbf{G})$ for each $i \in K_{\lambda}$. Further, we have $p_{\lambda}\left(f_{i}(y) ; i \in K_{\lambda}\right)=f(y)$ and $p_{\lambda}\left(f_{i}\left(y_{j}\right) ; i \in K_{\lambda}\right)=f\left(y_{j}\right)$ for each $j \in K_{\mu}$ (by the definition of the direct product of partial algebras). Thus, $M$ is $p_{\lambda} p_{\mu}$-operational with $p_{\lambda} p_{\mu}(M)=f(y)$ and $M^{T}$ is $p_{\lambda}$-operational. Since $\mathbf{G}$ is commutative, we have $p_{\mu} p_{\lambda}\left(M^{T}\right)=f(y)$. Thus, $p_{\lambda}\left(f\left(y_{j}\right) ; j \in K_{\mu}\right)=f(y)$, which yields $f \in \operatorname{Hom}(\mathbf{H}, \mathbf{G})$.

Corolla RY 3.10. Let $\mathbf{G}, \mathbf{H}$ be partial algebras of the same type. If $\mathbf{G}$ is both diagonal and commutative and $\mathbf{H}$ is idempotent, then the power $\mathbf{G}^{\mathbf{H}}$ is a partial subalgebra of the direct product $\mathbf{G}^{|\mathbf{H}|}$. 
Pr o o f. Let $\mathbf{H}=\left\langle H,\left(q_{\lambda} ; \lambda \in \Omega\right)\right\rangle, \mathbf{G}=\left\langle G,\left(p_{\lambda} ; \lambda \in \Omega\right)\right\rangle, \mathbf{G}^{\mathbf{H}}=\langle\operatorname{Hom}(\mathbf{H}, \mathbf{G})$, $\left.\left(r_{\lambda} ; \lambda \in \Omega\right)\right\rangle, \mathbf{G}^{H}=\left\langle G^{H},\left(R_{\lambda} ; \lambda \in \Omega\right)\right\rangle$ and let $\mathbf{G}$ be both diagonal and commutative and $\mathbf{H}$ be idempotent. Let $\lambda \in \Omega,\left(f_{i} ; i \in K_{\lambda}\right) \in D_{R_{\lambda}}, R_{\lambda}\left(f_{i}\right.$; $\left.i \in K_{\lambda}\right)=f$ and let $\left(y_{i} ; i \in K_{\lambda}\right) \in D_{q_{\lambda}}, q_{\lambda}\left(y_{i} ; i \in K_{\lambda}\right)=y$. Consider the $K_{\lambda} \times K_{\lambda}$-matrix $M=\left(f_{i j}\left(y_{i}\right) ; i \in K_{\lambda}, j \in K_{\lambda}\right)$ over $G$. As $p_{\lambda}\left(f_{j}\left(y_{i}\right)\right.$; $\left.j \in K_{\lambda}\right)=f\left(y_{i}\right)$ for each $i \in K_{\lambda}, M$ is $p_{\lambda}$-operational. By Theorem 3.9, $f \in \operatorname{Hom}(\mathbf{H}, \mathbf{G})$, which yields $p_{\lambda}\left(f\left(y_{i}\right) ; i \in K_{\lambda}\right)=f(y)$. Thus, $M$ is $p_{\lambda} p_{\lambda}$-operational with $p_{\lambda} p_{\lambda}(M)=f(y)$. Since $G$ is diagonal, $M$ is $p_{\lambda}$-operational with $\triangle p_{\lambda}(M)=f(y)$. Hence, $p_{\lambda}\left(f_{i}\left(y_{i}\right) ; i \in K_{\lambda}\right)=f(y)$. The idempotency of $\mathbf{H}$ implies the uniqueness of $f$. Therefore, $r_{\lambda}\left(f_{i} ; i \in K_{\lambda}\right)=f$.

Conversely, let $\left(f_{i} ; i \in K_{\lambda}\right) \in D_{r_{\lambda}}, r_{\lambda}\left(f_{i} ; i \in K_{\lambda}\right)=f$. Then $f \in \operatorname{Hom}(\mathbf{H}, \mathbf{G})$ and, since $\mathbf{H}$ is idempotent, we have $p_{\lambda}\left(f_{i}(x) ; i \in K_{\lambda}\right)=f(x)$ for each $x \in K_{\lambda}$. Hence, $R_{\lambda}\left(f_{i} ; i \in K_{\lambda}\right)=f$.

A partial algebra $\left\langle G,\left(p_{\lambda} ; \lambda \in \Omega\right)\right\rangle$ is said to be a (total) algebra provided that the domain $D_{p_{\lambda}}$ equals $G$ for every $\lambda \in \Omega$. If $\mathbf{G}$ and $\mathbf{H}$ are algebras of the same type, then the power $\mathbf{G}^{\mathbf{H}}$ need not be an algebra even if $\mathbf{H}$ is idempotent - see the following example:

Example 3.11. Let $\mathbf{H}=(H, q), \mathbf{G}=(G, p)$ be algebras of type (2) where $H=\{1,2\}, G=\{a, b\}$ and the binary oparations $q$ and $p$ are given by the tables

\begin{tabular}{|c|cc|}
\hline$q$ & 1 & 2 \\
\hline 1 & 1 & 2 \\
2 & 2 & 2 \\
\hline
\end{tabular}

and

\begin{tabular}{|l|ll|}
\hline$p$ & $\mathrm{a}$ & $\mathrm{b}$ \\
\hline $\mathrm{a}$ & $\mathrm{a}$ & $\mathrm{a}$ \\
$\mathrm{b}$ & $\mathrm{a}$ & $\mathrm{b}$ \\
\hline
\end{tabular}

Clearly, $\mathbf{H}$ is idempotent. Let $f: H \rightarrow G$ be the map given by $f(1)=a$, $f(2)=a, g: H \rightarrow G$ be the map given by $g(1)=b, g(2)=b$ and $h: H \rightarrow G$ be the map given by $h(1)=b, h(2)=a$. Then $\operatorname{Hom}(\mathbf{H}, \mathbf{G})=\{f, g, h\}$. Let $\mathbf{G}^{\mathbf{H}}=(\operatorname{Hom}(\mathbf{H}, \mathbf{G}), r)$. Then we have $r(g, h) \neq g, r(g, h) \neq h$ and $r(g, h) \neq f$, so $(g, h) \notin D_{r}$. Thus, $D_{r} \neq(\operatorname{Hom}(\mathbf{H}, \mathbf{G}))^{2}$, which implies that $\mathbf{G}^{\mathbf{H}}$ is not a (total) algebra.

It is evident that, for algebras, the diagonality and strong diagonality coincide and, for commutative algebras, also the diagonality and weak diagonality coincide. By Corollary 3.10, given algebras $\mathbf{G}$ and $\mathbf{H}$ of the same type, $\mathbf{G}^{\mathbf{H}}$ is an algebra whenever $\mathbf{G}$ is commutative and diagonal and $\mathbf{H}$ is idempotent (because the direct product of a family of algebras of the same type is an algebra, and a partial subalgebra of an algebra is an algebra too). Thus, by Theorem 3.4 we get: 
Corollary 3.12. Let $\mathbf{G}, \mathbf{H}$ be algebras of the same type, $\mathbf{H}$ idempotent. If $\mathbf{G}$ is both diagonal and commutative, then so is the algebra $\mathbf{G}^{\mathbf{H}}$.

Further, as a consequence of Theorem 3.11, we get:

TheOrem 3.13. Let $\mathbf{G}, \mathbf{H}, \mathbf{K}$ be algebras of the same type. If $\mathbf{G}$ is both diagonal and commutative and $\mathbf{H}, \mathbf{K}$ are idempotent, then $\left(\mathbf{G}^{\mathbf{H}}\right)^{\mathbf{K}}$ and $\mathbf{G}^{\mathbf{H} \times \mathbf{K}}$ are isomorphic algebras.

\section{Remark 3.14.}

(a) Clearly, for algebras with just one operation, diagonality implies commutativity. The idempotent and diagonal algebras with just one finitary operation were studied by J. Plonka in 5. He showed that these algebras are, up to isomorphisms, the algebras $\left(X_{1} \times \cdots \times X_{n}, p\right)$ where $n$ a positive integer, $X_{1}, \ldots, X_{n}$ are sets and the operation $p:\left(X_{1} \times \cdots \times X_{n}\right)^{n} \rightarrow X_{1} \times \cdots \times X_{n}$ is given by $p\left(\left(x_{1}^{1}, \ldots, x_{n}^{1}\right), \ldots,\left(x_{1}^{n}, \ldots, x_{n}^{n}\right)\right)=\left(x_{1}^{1}, x_{2}^{2}, \ldots, x_{n}^{n}\right)$.

(b) Commutative groupoids are usually called medial — see [3] where these groupoids are studied. Clearly, a groupoid $\mathbf{G}=(G, \cdot)$ is diagonal if and only if it is a semigroup with $x y z=x z$ whenever $x, y, z \in G$. Idempotent and diagonal groupoids are caled rectangular bands.

(c) Recall that a groupoid with a neutral element is called a loop provided that it satisfies both the left and right unique division laws. By [4, a loop $\mathbf{G}$ is medial if and only if it has the so-called Hamiltonian property (i.e., each subloop of $\mathbf{G}$ is a block of a congruence on $\mathbf{G}$ ).

(d) It may easily be seen that a diagonal algebra is locally diagonal if and only if it is idempotent. It is also evident that, for diagonal algebras, local antidiagonality is equivalent to weak local antidiagonality. Since every diagonal algebra $\left\langle G,\left(p_{\lambda} ; \lambda \in \Omega\right)\right\rangle$ has a $p_{\lambda}$-idempotent for any $\lambda \in \Omega$, namely, an arbitrary element $p_{\lambda}\left(x_{i} ; i \in K_{\lambda}\right)$ with $x_{i}=x$ ( $x \in G$ an element) for all $i \in K_{\lambda}$, a diagonal algebra $\left\langle G,\left(p_{\lambda} ; \lambda \in \Omega\right)\right\rangle$ is locally antidiagonal if and only if card $G=1$ or card $K_{\lambda}=1$ for every $\lambda \in \Omega$. Therefore, no interesting results may be obtained concerning the stability of the "local" properties of algebras under their powers.

\section{REFERENCES}

[1] BIRKHOFF, G.: Generalized arithmetic, Duke Math. J. 9 (1942), 283-302.

[2] BURMEISTER, P.: A Model Theoretic Oriented Approach to Partial Algebras, AcademieVerlag, Berlin, 1986.

[3] JEŽEK, J.-KEPKA, T.: Medial Groupoids, Rozpravy ČSAV, Řada Mat. Přír. Věd. 93, Academia, Prague, 1983. 


\section{N. CHAISANSUK - S. LEERATANAVALEE - J. ŠLAPAL}

[4] KLUKOVITS, L.: On commuative algebras, Acta Sci. Math. (Szeged) 34 (1973), 171-174.

[5] PLONKA, J.: Diagonal algebras, Fund. Math. 58 (1966), 309-321.

[6] ŠLAPAL, J.: Direct arithmetic of relational systems, Publ. Math. Debrecen 38 (1991), 39-48.

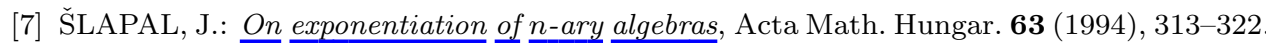

[8] ŠLAPAL, J.: Exponentiality in categories of partial algebras, Math. Pannon. 13 (2002), 201-206.

[9] S̆LAPAL, J.: Another cartesian closed category of partial algebras, Demonstratio Math. 4 (2000), 679-694.

Received 16. 12. 2011

Accepted 15. 2. 2012

\author{
* Department of Mathematics \\ Chiang Mai University \\ Faculty of Science \\ Chiang Mai \\ THAILAND \\ E-mail: nitimachaisansuk@gmail.com \\ sorasak.1@cmu.ac.th \\ ** Institute of Mathematics \\ Brno University of Technology \\ CZ-616 69 Brno \\ CZECH REPUBLIC \\ E-mail: slapal@fme.vutbr.cz
}

